\title{
Filamentary pattern in the cosmic web: galaxy filaments as pearl necklaces
}

\author{
Elmo Tempel ${ }^{1,2}$ and Maarja Bussov ${ }^{1}$ \\ ${ }^{1}$ Tartu Observatory, Observatooriumi 1, 61602 Tõravere, Estonia \\ email: elmo.tempel@to.ee \\ ${ }^{2}$ NICPB, Rävala pst 10, 10143 Tallinn, Estonia
}

\begin{abstract}
Galaxies form chains (filaments) that connect groups and clusters of galaxies. The filamentary network includes nearly half of the galaxies and is visually the most striking feature in cosmological maps. We study the distribution of galaxies along such a filamentary network, trying to find specific patterns. Our galaxy filaments are defined using the Bisous process. We use the two-point correlation function and the Rayleigh $Z$-squared statistic to study how the galaxies are distributed along the filaments. We show that galaxies and galaxy groups are not uniformly distributed along filaments, but tend to form a regular pattern. The characteristic length of the pattern is $7 h^{-1} \mathrm{Mpc}$. A slightly smaller characteristic length $4 h^{-1} \mathrm{Mpc}$ can also be found, using the $Z$-squared statistic. One can say that galaxy filaments are like pearl necklaces, where the pearls are galaxy groups distributed more or less regularly along the filaments. We propose that this well defined characteristic scale could be used as a cosmological test.
\end{abstract}

Keywords. Methods: numerical - methods: observational - large-scale structure of Universe.

\section{Introduction}

It is well known that galaxy filaments are visually the most dominant structures in the galaxy distribution, being part of the so-called cosmic network. Presumably, nearly half $(\sim 40 \%)$ of the galaxies (or mass in simulations) are located in filaments (Jasche et al. 2010; Cautun et al. 2014). Tempel et al. (2014a) shows that filaments extracted from the spatial distribution of galaxies/haloes are also dynamical structures that are well connected with the underlying velocity field. Galaxy filaments that connect groups and clusters of galaxies are also affecting the evolution of galaxies (Tempel \& Libeskind 2013; Zhang et al. 2013).

In this paper we study the distribution of galaxies along galaxy filaments to search for regularities in galaxy and group distributions. Such a clustering pattern exists at least in some filaments as shown decades ago (e.g. Jõeveer et al. 1978; Einasto et al. 1980). In the current study, we use the two-point correlation function and the Rayleigh $Z$-squared statistics to look for the regularity in the galaxy distribution. We suggest that the regularities we find in the galaxy distribution could be used as cosmological probes for dark energy and dark matter.

\section{Data and methods}

The present work is based on the SDSS DR10. We use the galaxy and group samples as compiled in Tempel et al. (2014b) that cover the main contiguous area of the survey (the Legacy Survey, approximately $17.5 \%$ from the full sky). The flux-limited catalogue extends to the redshift $0.2\left(574 h^{-1} \mathrm{Mpc}\right)$ and includes 588193 galaxies and 82458 groups with two or more members. 

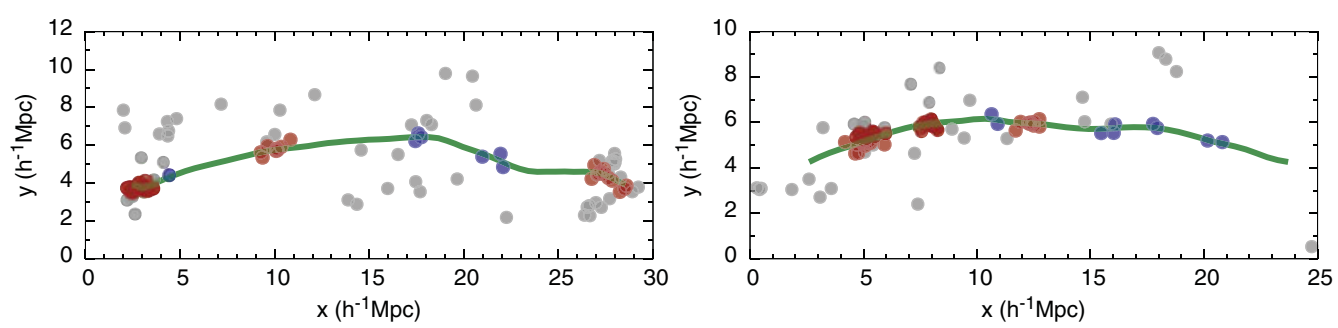

Figure 1. Examples of filaments and their spines. Red points show galaxies in filaments (closer than $0.5 h^{-1} \mathrm{Mpc}$ to the filament axis) that are located in groups with 5 or more members. Blue points show other galaxies in filaments. Grey points are background galaxies that are not located in these filaments. The thick green line shows the spine of a filament.

In Tempel et al. (2014b) the redshift-space distortions, the so-called finger-of-god (FoG) effects, are suppressed using the rms sizes of galaxy groups in the plane of the sky and their rms radial velocities as described in Liivamägi et al. (2012). We calculate the new radial distances for galaxies in groups in order to make the galaxy distribution in groups approximately spherical. We note that such a compression will remove the artificial lineof-sight filament-like structures.

The detection of filaments is performed by applying an object/marked point process with interactions (the Bisous process) to the distribution of galaxies. This algorithm provides a quantitative classification which agrees with the visual impression of the cosmic web and is based on a robust and well-defined mathematical scheme. A detailed description of the Bisous model is given in Stoica et al. (2007, 2010) and Tempel et al. (2014c). For reader convenience, a brief and intuitive description is given below.

The marked point process we propose for filament detection is different from the ones already used in cosmology. In fact, we do not model galaxies, but the structure outlined by galaxy positions.

This model approximates the filamentary network by a random configuration of small segments (thin cylinders). We assume that locally galaxies may be grouped together inside a rather small cylinder, and such cylinders may combine to form a filament if neighbouring cylinders are aligned in similar directions. This approach has the advantage that it is using only positions of galaxies and does not require any additional smoothing to create a continuous density field.

The solution provided by our model is stochastic. Therefore, we find some variation in the detected patterns for different MCMC runs of the model. The main advantage of using such a stochastic approach is the ability to give simultaneous morphological and statistical characterisation of the filamentary pattern.

In practice, after fixing the approximate scale of the filaments, the algorithm returns the filament detection probability field together with the filament orientation field. Based on these data, filament spines are extracted and a filament catalogue is built. Every filament in this catalogue is represented as a spine: a set of points that define the axis of the filament.

The spine detection we use is based on two ideas. First, filament spines are located at the highest density regions outlined by the filament probability maps. Second, in these regions of high probability for the filamentary network, the spines are oriented along the orientation field of the filamentary network.

The filaments are extracted from a flux-limited galaxy sample, hence, the completeness of extracted filaments decreases with distance. In Tempel et al. (2014c) we showed that the volume filling fraction of filaments is roughly constant with distance if filaments 
longer than $15 h^{-1} \mathrm{Mpc}$ are considered. Therefore, in the current study we are using only filaments longer than this limit. In addition, this choice is justified, since longer filaments allow us to study the distribution of galaxies along the filaments, which is the purpose of this paper. The remaining incompleteness of filaments in our sample is not a problem, because we are analysing single filaments. The filaments we use are the strongest filaments (or segments of filaments) in the sample.

To suppress the flux-limited sample effect, we volume-limit the galaxy content for single filaments. For that, we find for every filament the maximum distance (from the observer) of its galaxies and the corresponding magnitude limit and use only galaxies brighter than that. Since the majority of filaments extend over a relatively narrow distance interval, the number of excluded galaxies in every filament is small.

To study the galaxy spacing along the filaments, we project every galaxy to the filament spine. Hence, the distance is measured along the filament spine. Figure 1 illustrates extracted galaxy filaments and their spines in the field of galaxies. For our analysis, we use only filaments that contain at least 10 galaxies. When studying galaxy groups, we use only filaments where the number of groups per filament is at least 5 .

To study galaxy correlations in filaments, we use the two-point correlation function $\xi(\mathbf{r})$ that measures the excess probability of finding two points separated by a vector $\mathbf{r}$ compared to that probability in a homogeneous Poisson sample. For galaxy filaments, the correlation function along the filament can be expressed in terms of the distances between the galaxies measured along the filament.

We estimate $\xi(r)$ following the Landy-Szalay border-corrected estimator (Landy \& Szalay 1993). We generated a random distribution of points for each filament considered, and estimated the correlation function $\xi(r)$ :

$$
\widehat{\xi}(r)=1+\frac{D D(r)}{R R(r)}-2 \frac{D R(r)}{R R(r)},
$$

where $D D(r), R R(r)$, and $D R(r)$ are the probability densities for the galaxy-galaxy, random-random, and galaxy-random pairs, respectively, for a pair distance $r$.

Using Eq. (2.1), we estimate the correlation function for each filament separately. The final pair correlation function, averaged over all filaments, is estimated as a sum over all filaments. We estimate the statistical error on our $\xi^{\text {fil }}(r)$ measurements with the standard jackknife method, where we omit one filament under consideration at a time. In our figures we show the $95 \%$ confidence intervals.

To test whether the galaxy distribution might have regularity along the filaments we use the Rayleigh (or $Z$-squared) statistic. It is an excellent method when the event rate (in our case, the number of galaxies per filament) is low. The method has been used to detect periodicity in time series for the data in the form of discrete events (photon arrival times) and can be applied to detect periodicity in the galaxy distribution along filaments, where the galaxy positions can be considered as events.

The algorithm works as follows. For each filament, we produce a periodogram using the $Z_{1}^{2}$ (Rayleigh statistic),

$$
Z_{1}^{2}=\frac{2}{N}\left[\left(\sum_{j=1}^{N} \cos \phi_{j}\right)^{2}+\left(\sum_{j=1}^{N} \sin \phi_{j}\right)^{2}\right],
$$

where $N$ is the number of galaxies in a filament and $\phi_{j}=2 \pi l_{j} / d$ is the phase value for a galaxy $j$ for a fixed period $d ; l_{j}$ is a distance of the galaxy $j$ along the filament spine from the beginning of the filament. 

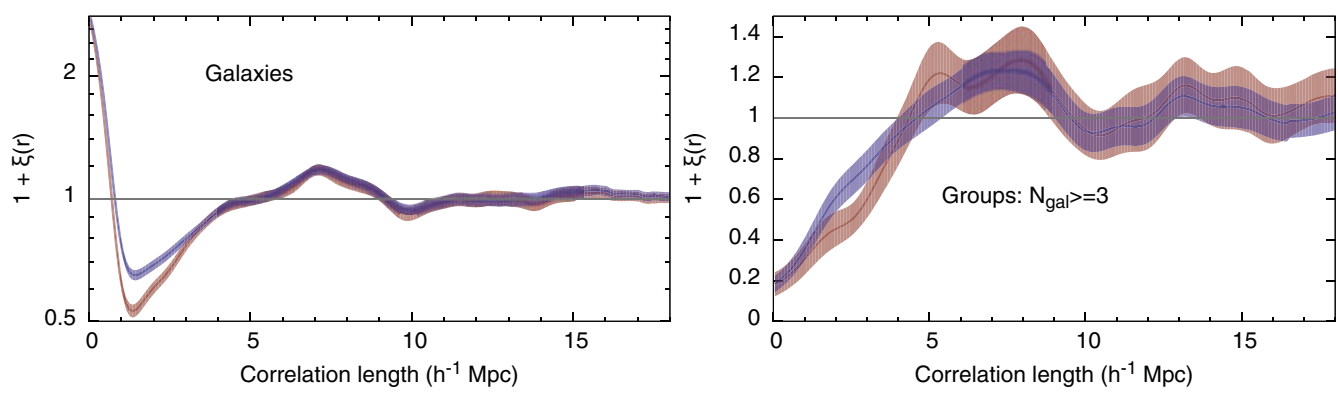

Figure 2. Galaxy (left panel) and group (right panel) correlation function along filaments. Blue and red lines with filled regions show the correlation function together with its $95 \%$ confidence limits (based on a jackknife estimate) for galaxies/groups closer than 0.5 and $0.25 h^{-1} \mathrm{Mpc}$ to the filament axis, respectively.

To measure the $Z$-squared statistic for a period $d$, we are using only filaments longer than $2 d$. This assures that there are at least two periods for each filament. We are deriving a null-hypothesis probability function using Monte Carlo simulations with $N$ (the number of galaxies in a filament) data points assuming an uniform distribution of points along the spine of the filament. This allows us also to estimate the confidence intervals for our measured signal.

We compute the $Z_{1}^{2}$ statistic as a function of a period $d$ for every filament and then we find the average signal using the filament length $L_{\mathrm{fil}}$ as the weight.

\section{Results and discussion}

Figure 2 (left panel) shows the galaxy correlation function along filaments. Three specific features are seen in this correlation function: a maximum near the zero pair distance, a minimum that follows it, and a bump close to $7 h^{-1} \mathrm{Mpc}$. The first maximum is caused by galaxy groups. It shows that galaxies are not distributed uniformly in the space, they form groups and clusters, as it is well known. The minimum next to the first maximum shows that groups themselves are not distributed uniformly along filaments. It shows that two groups cannot be located directly close to each other (merging groups are exceptions) and there exists some preferred minimum distance between galaxy groups. This is also expected since matter is falling into groups and there is not enough matter in a close-by neighbourhood of groups to form another group. The most interesting feature is the small maximum close to $7 h^{-1} \mathrm{Mpc}$. It shows that galaxies (and also groups) have a tendency to be separated by this distance along the filaments.

In Fig. 2 (right panel) we show the correlation function for galaxy groups with at least three members. In this figure, the smoothing scale is twice as large as used for the galaxy correlation functions and we analyse only filaments that contain at least five groups. We see that the bump around $7 h^{-1} \mathrm{Mpc}$ is present also for groups, indicating that groups themselves have some preferred distance between them. We analysed groups with different minimum group richness (the number of galaxies in groups). The $7 h^{-1} \mathrm{Mpc}$ bump was higher if isolated galaxies $\left(N_{\text {gal }}=1\right)$ were excluded.

The biggest difference when comparing the correlation function for galaxies (left panel in Fig. 2) and for groups (right panel in Fig. 2) is the fact that there is no zero-distance maximum for groups. This shows that groups themselves do not form clusters, which is expected. The minimum close to zero distance in the group correlation function shows that there is a lack of other groups around each group. The radius of the group influence extends to $4 h^{-1} \mathrm{Mpc}$, after that the correlation function is mostly flat. 

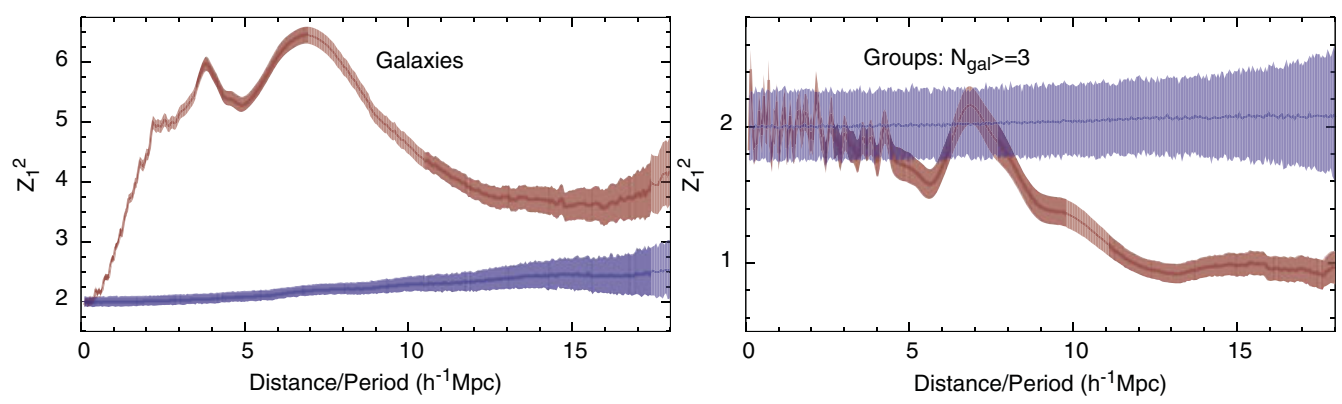

Figure 3. The dependence of the Rayleigh ( $Z$-squared) statistic $Z_{1}^{2}$ on distance (period). The left panel shows the results for galaxies closer than $0.5 h^{-1} \mathrm{Mpc}$ to the filament axis and right panel shows the results for galaxy groups. The red line shows the $Z_{1}^{2}$ statistic together with the jackknife $95 \%$ confidence estimate. The blue line shows the results from Monte Carlo simulations for the null hypothesis together with the $95 \%$ confidence limits.

Figure 3 (left panel) shows the $Z$-squared statistic for all galaxies closer than $0.5 h^{-1} \mathrm{Mpc}$ to the filament axis. The $Z$-squared statistic based on galaxies is shown with red lines, where the shaded region shows the $95 \%$ confidence limits. The blue line shows the statistic for the null hypothesis using Monte Carlo simulation for a Poisson sample. The shaded region shows the $95 \%$ confidence limits for this case. For Monte Carlo simulation, the filaments and numbers of galaxies per filament are the same as for the real sample, but galaxies are Poisson distributed. Since we are averaging filaments with different lengths, the $Z$-squared statistic for the Poisson sample is not exactly around 2 as predicted by theory. Since the deviation is small, it does not affect our conclusions.

The maximum around $7 h^{-1} \mathrm{Mpc}$ that was visible in the correlation functions is also visible in Fig. 3, confirming that galaxies are distributed along filaments in some regular pattern. Interestingly, the $Z_{1}^{2}$ statistic shows that there is also a small maximum around $4 h^{-1} \mathrm{Mpc}$. This indicates that between two groups that are separated by $7 h^{-1} \mathrm{Mpc}$ there is quite often another group.

Figure 3 (right panel) shows the $Z$-squared statistic for groups with three or more member galaxies. The blue region shows the Monte Carlo simulation results for a Poisson sample. The $Z_{1}^{2}$ statistic for groups lies considerably below of that for the Poisson sample. This indicates that galaxy groups are distributed along filaments more regularly than in the Poisson case. This is expected, since galaxy groups cannot be located directly close to each other, there is some minimum distance between the groups that is also visible in the two-point correlation functions. We analysed groups with different minimum group richness. The $7 h^{-1} \mathrm{Mpc}$ scale was the strongest for groups with at least three member galaxies. It shows that the regularity is stronger if the weakest groups are excluded.

In Fig. 3 we see that the maxima around 4 and $7 h^{-1} \mathrm{Mpc}$ that define the preferred scales for the galaxy distribution, are also visible in the group distributions. This indicates that it is related to galaxy clustering.

We refer to Tempel et al. (2014d) for more detailed analysis of the results presented here.

\section{Conclusions}

Using the Bisous model (marked point process with interactions) we extracted the galaxy filaments from the SDSS spectroscopic galaxy survey. The diameter of the extracted filaments is roughly $1 h^{-1} \mathrm{Mpc}$ and the catalogue of filament spines is built as described in Tempel et al. (2014c). Using the galaxies and groups in filaments (with a 
distance from the filament axis less than $0.5 h^{-1} \mathrm{Mpc}$ ) we studied how the galaxies/groups are distributed along the filament axis. The main results of our study can be summarised as following.

- The galaxy and group distributions along filaments show a regular pattern with a preferred scale around $7 h^{-1} \mathrm{Mpc}$. A weaker regularity is also visible at a scale of $4 h^{-1} \mathrm{Mpc}$. The regularity of the distribution of galaxies along filaments is a new result that might help to understand structure formation in the Universe.

- The pair correlation functions of galaxies and groups along filaments show that around each group, there is a region where the number density of galaxies/groups is smaller than on average.

- Galaxy groups in the Universe are more uniformly distributed along filaments than in the Poisson case.

The clustering pattern of galaxies and groups along filaments tells us that galaxy filaments are like pearl necklaces, where the pearls are galaxy groups that are distributed along the filaments in some regular pattern.

We suggest that the measured regularity of the galaxy distribution along filaments could be used as a cosmological probe to discriminate between various dark energy and dark matter cosmological models. Additionally, it can be used to probe environmental effects in the formation and evolution of galaxies. We plan to test these hypothesis in our following analysis using N-body simulations and deep redshift surveys like the Galaxy And Mass Assembly (GAMA) and the VIMOS Public Extragalactic Survey (VIPERS).

Acknowledgements. This work was supported by ESF grants/projects MJD272, IUT40-2, TK120. We thank SDSS for the publicly available data releases.

\section{References}

Cautun, M., van de Weygaert, R., Jones, B. J. T., \& Frenk, C. S. 2014, MNRAS, 441, 2923

Einasto, J., Jõeveer, M., \& Saar, E. 1980, Nature, 283, 47

Jasche, J., Kitaura, F. S., Li, C., \& Enßlin, T. A. 2010, MNRAS, 409, 355

Jõeveer, M., Einasto, J., \& Tago, E. 1978, MNRAS, 185, 357

Landy, S. D. \& Szalay, A. S. 1993, ApJ, 412, 64

Liivamägi, L. J., Tempel, E., \& Saar, E. 2012, A\&SA, 539, A80

Stoica, R. S., Martínez, V. J., \& Saar, E. 2007, Journal of the Royal Statistical Society Series $C, 56,459$

Stoica, R. S., Martínez, V. J., \& Saar, E. 2010, A\& $A$, 510, A38

Tempel, E. \& Libeskind, N. I. 2013, ApJL, 775, L42

Tempel, E., Libeskind, N. I., Hoffman, Y., Liivamägi, L. J., \& Tamm, A. 2014a, MNRAS, 437, L11

Tempel, E., Tamm, A., Gramann, M., Tuvikene, T., Liivamägi, L. J., Suhhonenko, I., Kipper, R., Einasto, M., \& Saar, E. 2014b, A\&A, 566, A1

Tempel, E., Stoica, R. S., Martínez, V. J., Liivamägi, L. J., Castellan, G., \& Saar, E. 2014c, MNRAS, 438, 3465

Tempel, E., Kipper, R., Saar, E., Bussov, M., Hektor, A., \& Pelt, J. 2014d, arXiv:1406.4357

Zhang, Y., Dietrich, J. P., McKay, T. A., Sheldon, E. S., \& Nguyen, A. T. Q. 2013, ApJ, 773, 115 